\title{
Participative Enterprise Modeling: Experiences and Recommendations
}

\author{
Janis Stirna $^{1}$, Anne Persson ${ }^{2}$, and Kurt Sandkuhl ${ }^{1}$ \\ ${ }^{1}$ Jönköping University, PO Box 1026, SE-551 11, Jönköping, Sweden \\ \{janis.stirna, kurt. sandkuhl\} @ing.hj.se \\ ${ }^{2}$ University of Skövde, P.O. Box 408, SE-541 28 Skövde, Sweden \\ anne.persson@his.se
}

\begin{abstract}
The objective of this paper is to report a set of experiences of applying participative enterprise modeling in different organizational contexts. While the authors have successfully applied the approach in many organizations, the paper primarily concentrates on three cases. On the basis of these experiences the paper presents a set of generic principles for applying participative enterprise modeling.
\end{abstract}

Keywords: Enterprise modeling, participative modeling.

\section{Introduction}

Enterprise Modeling (EM) is an activity where an integrated and commonly shared model describing different aspects of an enterprise is created. An Enterprise Model comprises a number of related "sub-models", each focusing on a particular aspect of the problem domain, such e.g. processes, business rules, concepts/information/data, vision/goals, and actors. EM is often used for developing organization's strategies, business process restructuring, business process orientation, communication of work procedures, eliciting information system requirements, etc. More about the applicability of EM is available in [1]. In all these activities the development team addresses frequent challenges of how to discover the domain knowledge, how to consolidate different stakeholder views, and how to represent this knowledge in a coherent and comprehensive model. Additionally, there is a need to ensure that the decisions made and reflected in the models are taken-up and implemented in reality. Addressing these challenges by the traditional consulting approach of fact gathering, analysis and then delivering an expert opinion is not efficient when dealing with "illstructured" or "wicked" problems [2] typically occurring in organizations. As a result participative EM, i.e. modeling in facilitated group sessions, has been established as a valuable and practicable instrument for solving organizational design problems (c.f., e.g. $[3,4,5,6,7])$.

Therefore, the objective of this paper is to report a set of experiences of applying participative enterprise modeling in different organizational contexts. While the approach has been successfully applied in many organizations this paper primarily concentrates on three cases, namely a healthcare organization, a firm developing 
components for the automotive industry, and a municipality. On the basis of these experiences we also present a set of generic participative EM principles.

The research approach is conceptual and argumentative based on a number of case studies that were carried out in public and private organizations $[8,9,10]$ and an interview study with experienced practitioners [11].

The remainder of the paper is organized as follows. In section 2 we provide a background to EM methods and ways of working. Section 3 presents three case studies, namely, Kongberg Automotive, the Riga City Council and Skaraborgs Sjukhus. Section 4 presents a set of best practices of participative EM. The recommendations are illustrated using the three cases. Conclusions and future work are, finally, discussed in section 5 .

\section{Background to Enterprise Modeling}

In Scandinavia, Business or Enterprise Modeling were initially developed in the eighties by Plandata, Sweden [12], and later refined by the Swedish Institute for System Development (SISU). A significant innovation in this strand of EM was the notion of business goals as part of an Enterprise Model, enriching traditional model component types such as entities, and business processes. The SISU framework was further developed in the ESPRIT projects F3 - "From Fuzzy to Formal" and ELEKTRA - "Electrical Enterprise Knowledge for Transforming Applications". The current framework is denoted EKD - "Enterprise Knowledge Development" [4, 6]. Apart from the "Scandinavian" strand of EM, a variety of other methods have been suggested (c.f., e.g. [13], [14], [15], [16], [17], [18]).

[1] show that EM can be used for two main types of objectives - (1) developing the business, e.g. developing business vision, strategies, redesigning the way the business operates, developing the supporting information systems, or (2) ensuring the quality of the business, e.g. sharing the knowledge about the business, its vision, the way it operates, or ensuring the acceptance of business decisions through committing the stake-holders to the decisions made.

\subsection{EKD}

EKD - Enterprise Knowledge Development method [6] is a representative of the Scandinavian strand of EM methods. It defines the modeling process as a set of guidelines for participative way of working and the modeling product in terms of six sub-models each focusing on a specific aspect of an organization (see table 1).

The ability to trace decisions, components and other aspects throughout the enterprise is dependent on the use and understanding of the relationships between the different sub-models addressing the issues in table 1. When developing a full enterprise model, these relationships between components of the different sub-models play an essential role. E.g. statements in GM allow different concepts to be defined more clearly in the CM. A link is then specified between the corresponding GM component and concepts in CM. In the same way, goals in the GM motivate the existence of processes in the BPM. Links between models make the model traceable. They show, for instance, why certain rules, processes and information system requirements have been introduced. 
Table 1. Overview of the sub-models of the EKD method

\begin{tabular}{|c|c|c|c|c|c|c|}
\hline & $\begin{array}{l}\text { Goals Model } \\
\text { (GM) }\end{array}$ & $\begin{array}{l}\text { Business } \\
\text { Rules Model } \\
\text { (BRM) }\end{array}$ & $\begin{array}{l}\text { Concepts } \\
\text { Model (CM) }\end{array}$ & $\begin{array}{l}\text { Business } \\
\text { Process Model } \\
\text { (BPM) }\end{array}$ & $\begin{array}{l}\text { Actors and } \\
\text { Resources } \\
\text { Model (ARM) }\end{array}$ & $\begin{array}{l}\text { Technical } \\
\text { Component \& } \\
\text { Requirements } \\
\text { Model(TCRM) }\end{array}$ \\
\hline Focus & $\begin{array}{l}\text { Vision and } \\
\text { strategy }\end{array}$ & $\begin{array}{l}\text { Policies and } \\
\text { rules }\end{array}$ & $\begin{array}{l}\text { Business } \\
\text { ontology }\end{array}$ & $\begin{array}{l}\text { Business } \\
\text { operations }\end{array}$ & $\begin{array}{l}\text { Organizational } \\
\text { structure }\end{array}$ & $\begin{array}{l}\text { Information } \\
\text { system needs }\end{array}$ \\
\hline Issues & $\begin{array}{l}\text { What does } \\
\text { the organi- } \\
\text { zation want } \\
\text { to achieve or } \\
\text { to avoid and } \\
\text { why? }\end{array}$ & $\begin{array}{l}\text { What are the } \\
\text { business } \\
\text { rules, how do } \\
\text { they support } \\
\text { organiza- } \\
\text { tion's goals? }\end{array}$ & $\begin{array}{l}\text { What are the } \\
\text { things and } \\
\text { "phenomena" } \\
\text { addressed in } \\
\text { other sub- } \\
\text { models? }\end{array}$ & $\begin{array}{l}\text { What are the } \\
\text { business } \\
\text { processes? How } \\
\text { do they handle } \\
\text { information and } \\
\text { material? }\end{array}$ & $\begin{array}{l}\text { Who are } \\
\text { responsible for } \\
\text { goals and } \\
\text { process? How } \\
\text { are the actors } \\
\text { interrelated? }\end{array}$ & $\begin{array}{l}\text { What are the } \\
\text { business } \\
\text { requirements to } \\
\text { the IS? How are } \\
\text { they related to } \\
\text { other models? }\end{array}$ \\
\hline $\begin{array}{l}\text { Com- } \\
\text { po- } \\
\text { nents }\end{array}$ & $\begin{array}{l}\text { Goal, prob- } \\
\text { lem, external } \\
\text { constraint, } \\
\text { opportunity }\end{array}$ & Business rule & $\begin{array}{l}\text { Concept, } \\
\text { attribute }\end{array}$ & $\begin{array}{l}\text { Process, } \\
\text { external proc., } \\
\text { information set, } \\
\text { material set }\end{array}$ & $\begin{array}{l}\text { Actor, role, } \\
\text { organizational } \\
\text { unit, individual }\end{array}$ & $\begin{array}{l}\text { IS goal, } \\
\text { IS problem, } \\
\text { IS requirement, } \\
\text { IS component }\end{array}$ \\
\hline
\end{tabular}

\subsection{C3S3P}

$\mathrm{C} 3 \mathrm{~S} 3 \mathrm{P}$ is based on work in several EU projects from the area of networked and extended enterprises. An extended enterprise is a dynamic networked organization, which is created ad-hoc to reach a certain objective using the resources of the participating cooperating enterprises. In order to support solutions development for extended enterprises, the EXTERNAL project [19] developed a methodology for extended enterprise modeling [20], which initially was named SGAMSIDOER based on the abbreviations of the different modeling steps proposed: Scoping of the extended enterprise, Gather existing partner information, Analyze extended enterprise potential, Model extended enterprise, Simulate and analyze model scenarios, Implement Model, Deploy extended enterprise, Operate, as well as Evaluate, Reengineer extended enterprise. This methodology was further developed towards a complete customer delivery process denoted C3S3P, which is used in the ATHENA ${ }^{1}$ and MAPPER ${ }^{2}$ projects. C3S3P, like SGAMSIDOER, aims at executable solutions based on visual EM. The seven C3S3P phases are:

- Concept Testing: pre-studies are performed to investigate whether EM is a suitable and accepted way of developing executable solutions for the networked enterprise

- Scaffolding aims at creating shared knowledge and understanding among the participants of the project about the scope and challenges of the project.

- Scenario Modeling: creation of executable models supporting the networked enterprise in the defined scope including all relevant dimensions required, like process, product, organization or IT-systems

- Solutions Modeling: refining the scenario model by integration personnel, product structures, document templates and IT systems required for using the enterprise model in an actual project

- Platform Configuration: configure the solution models for use in the networked or extended enterprise by connecting the enterprise model to the platform used

\footnotetext{
${ }^{1} \mathrm{http} / / / \mathrm{www}$.athena-ip.org/

${ }^{2} \mathrm{http} / / /$ mapper.troux.com/
} 
- Platform Delivery: encompasses the roll-out of model-configured solutions

- Performance Improvement by capturing indicators for process and product quality and using adequate management instruments.

\section{Cases of Applying Participative EM in Practice}

This section presents three EM application cases at Kongberg Automotive, the Riga City Council and Skaraborgs Sjukhus.

\subsection{Participative EM at Kongsberg Automotive (KA)}

Kongsberg Automotive is a first tier supplier to the worldwide automotive industry developing and manufacturing gearshift controls and seat comfort systems. EM at KA was performed during the EU FP6 project MAPPER, which aims at supporting collaboration between different actors in the automotive supply chain by using reconfigurable enterprise models and providing an infrastructure with collaboration services and for executing these models. The enterprise models developed follow the POPS* approach [21]; they include dimensions like processes, organization structures, product structures, IT-systems and any other dimension relevant for the modeling purpose in one single visual enterprise model.

The development of enterprise models roughly followed the C3S3P approach (section 2.2) and was developed in different phases. In this paper we focus on the scaffolding phase, aiming to create a joint understanding in the modeling team about current situation, challenges and way of working of the modeling team. The group consisted of 3 to 7 employees from KA representing different roles (material specialist, electrical engineer, product manager, etc.), 3 to 4 participants from research organizations and 1 or 2 experts from the developer of the modeling tool used. All modeling sessions added to 6 days of work for the team. During the first modeling workshop the team agreed to use the following roles:

- Process owner being responsible for establishing the modeling activity within the enterprise, selecting the right personnel resources, arranging meetings, etc.

- Facilitator providing expertise in using the selected modeling process and tool as well as providing supports the modeling process and model development by coaching the modelers. This role facilitates model construction and development.

- Modeling expert having in-depth knowledge in the modeling method and tools.

- Tool operator responsible for documenting the enterprise models in the computerized tool during the modeling process

- Domain expert providing knowledge about the domain under consideration, which is basis for modeling.

The work was done during joint modeling workshops with about 10 participants. We used the visual EM tool METIS ${ }^{3}$. All aspects of modeling were jointly performed in a model projected on a large screen. Each modeling workshop started with defining objectives of the session. The scaffolding focused on the case under discussion - the

\footnotetext{
${ }^{3}$ Information about the METIS product is available at http://www.trouxmetis.com/
} 
process of innovation at KA. The main task was to establish a process for creating new and innovative products in the advanced engineering department.

After the workshops, the produced models were consolidated by the facilitator. This task aimed at improving the visual structure of the model, completing textual descriptions of the model elements and identifying open questions, inconsistencies and needs for refinement. The workshops that followed always started with a walkthrough of the current model version, in order to update all partners about the current status, introduce changes that have been made as well as raise issues for discussion.

The use of a visual modeling language supported the participatory approach providing a means for instant discussion of the modeling results, checking accuracy, and correcting potential shortcomings. It also helped updating all partners on the current status after a period of offline work. Visual modeling was equally efficient and useful in the initial sessions devoted to brainstorming and in final modeling sessions used for refining models. While the participative approach might have appeared to consume a lot of resources it gave the desired result of stakeholder involvement and correctness of the model. With a facilitator and tool operator managing the modeling tool skillfully the delays for updating the model were rather small. We have to recognize that with increasing level of detail, the visual models became quite complex and large, which makes it difficult to provide paper-based versions for stakeholders preferring to work with paper printouts. In our case there was no such stakeholder, but in other projects this would have been a likely situation.

\subsection{Paticipative EM at the Riga City Council (RCC)}

Riga City Council (RCC) is a municipality responsible for administration of public affairs of the city of Riga. In the past ten years the RCC has developed a large amount of information systems supporting its various functions. However, these systems did not address the growing need for managing RCC's organizational knowledge and competence. To answer this challenge RCC participated in FP5 IST project "Hypermedia and Pattern Based Knowledge Management for Smart Organizations" with the objective of developing and adopting a system for collecting and disseminating knowledge regarding strategic issues of capital importance for decision making at different levels in the city's administration [10].

The initial phase of the project was devoted to setting the Knowledge Management (KM) strategy, KM processes, and requirements for $\mathrm{KM}$ systems. We used participative EM and the EKD method. The way of working consisted of:

- Interviews about the current state and future vision for KM with ca 50 high ranking politicians and managers working in various committees and departments of the RCC. The additional intangible effect of these interviews was increased awareness about and popularity of the project.

- Two modeling sessions with the top level management of the RCC in order to decide the KM vision and outline the KM adoption process

- Selecting three KM pilot applications - at the Riga Drug Abuse Prevention Centre, at the School Board of Riga City, and at the Traffic Department. 
- A series of modeling sessions in each pilot area targeting specific issues of these departments. In total the sessions at this stage added to ca 10 days of work for several modeling teams of 5-10 participants. Each session started with a review of the work previously done.

- One modeling session about integrating the pilot cases and developing overall KM processes for the RCC.

The roles of participants were similar to the ones described in section 3.1. During the modeling sessions we used a large plastic wall and post-it notes to document the model. This approach proved to be useful because it does not require the modeling participants to "channel" their input to the model through an operator of a computerized tool, which often slows down the creative process. After the modeling session the facilitator documented the resulting models in the Visio tool. At the final stages of the project the modelers refined models by using the tool directly.

The participants accepted the participative way of working because it was apparent to them that it helps them to discuss issues openly and to agree on decisions. The role of facilitator was appreciated. In the beginning of the project the method providers acted as modeling facilitators, but as the project progressed the two people from the RCC developed the competency of facilitation and took over this role.

\subsection{Participative EM at Skaraborgs Sjukhus (SKaS)}

Skaraborgs Sjukhus (SKaS) is a cluster of hospitals in Western Sweden working together with primary care centers and municipal home care to provide high-quality healthcare to the citizens in the region within which they act. Some medical specialties have a higher degree of collaboration between the hospital, primary care centers and municipalities than others. An example of this is the treatment and prevention of leg ulcers. To decrease the healing time for various types of leg ulcers e.g. with diabetic patients large efforts are made by all actors involved to e.g. develop new and more efficient treatment methods and care routines. To address the challenge of efficient knowledge sharing among various actors in the healthcare process (e.g. nurses in primary care and municipal home care) all three healthcare organizations participated in a project to build a knowledge repository for learning and sharing of best practices with regard to treatment and prevention methods for leg ulcers [8].

In the project, participative EM has mainly been used as a means to develop a knowledge map that describes the content and structure of the knowledge repository. The knowledge map is in the form of an EKD concepts model. The roles of participants were similar to the ones described in section 3.1 and 3.2. The domain experts in this case were doctors and specialist nurses at the hospital. An initial version of the model was developed early on in the project using the "plastic wall" approach described in section 3.2. iGrafx Flowcharter was used to document the model. Throughout the project the model was refined a number of times as the understanding of the problem domain improved, among all actors involved. Changes to the model were made directly in the computer tool. In this case the concepts model functioned as a detailed "blueprint" for creating the knowledge repository. Therefore, particular attention was given to developing a model that was precise and correct. 


\section{Recommendations of Using Participative EM}

This section presents our recommendations for conducting participative EM in practice. While in the course of discussion we will refer to the three application cases outlined in section 3, the knowledge is also grounded into application cases in organizations such as e.g. British Aerospace (UK), Capital Bank (UK), Public Power Corporation (Greece), Sema Group (France), Telia (Sweden), Vattenfall (Sweden), Volvo (Sweden), Verbundplan (Austria), RRC College (Latvia). It is also built on an interview study mainly targeting experienced practitioners [11].

\subsection{Assess the Organizational Context}

In any project, understanding the organization's power and decision-making structure is essential. It is within the boundaries of these structures that the stakeholders create their Enterprise Model. Having access to and having the trust of the relevant decisionmakers is especially critical for participative EM project managers when it comes to obtaining enough effort from domain experts. The planning of participative EM sessions will openly reveal that the stakeholders involved will need to allocate their time and effort to modeling work. If participative EM is relatively new to the organization, the amount of man-hours will seem unnecessarily large, which may cause some reluctance with decision-makers. If other activities in the organization at any time are given a higher priority than the EM project, the resources allocated for modeling sessions will most likely be reduced. This will have a strong negative impact on the modeling result.

In the SKaS case the project had high priority with management and this priority was not changed during the project. Management also seemed to trust the judgments of project management. The group of domain experts and method experts was stable for the duration of the project. Certain days were allocated for the project in the domain experts' weekly schedules, which made it easy to plan for modeling sessions.

Organizational culture has significant impact on the results and effects of participative EM [1, 11]. In fact, it seems that failure to properly understand the culture of an organization is perhaps one of the most critical risks in participative EM. Participative EM requires that the participants consider themselves authorized to state their opinions and to suggest solutions. This approach is therefore only suitable in consensus-oriented organizations. In authoritative cultures, it will be extremely difficult to achieve consensus-driven participation in the modeling groups.

Official documents/systems (policy documents, strategy documents, internal instructions, web-site etc.) often reveal some of the organizational culture. Ask direct questions about how the organization looks upon the concepts of responsibility, cooperation and participation. This will give an idea of the management philosophy in the organization. Also, ask questions about how people in the organization will be informed about the project. If very strong restrictions are put on the involvement of a circle of people outside the modeling group, this may either indicate an authoritative culture or a hidden agenda. Ask questions about how any additional stakeholders may be contacted and involved. A strong enforcement of the official decision-making structure indicates that the modeling team will not be free to contact people without talking to their superiors. This may also indicate an authoritative culture. 
Attitudes towards participation are often revealed in the way people talk about the problem at hand, other people in the organization, etc. Observe how people act when talking with each other and with the method provider. Look for attitudes towards different types of actors (superiors, subordinates, opposite sex, etc.). E.g. in a group of people, it can be observed by looking at the faces of people whether or not they agree with what is said or whether they approve of another person or not. Exaggerated agreement with a superior may indicate a need to always express opinions that are in line with those of a superior. This may also indicate an authoritative culture.

In a consensus-oriented culture subordinates can question superiors, the dialogue between levels of the organization is open and direct and reward systems encourage initiatives from all levels of the organization. In an authoritative culture management is by directives only, the dialogue is indirect, and where there are no reward systems for initiatives from different levels of the organization. Note that in an organization, different types of cultures can reside in departments, divisions, subsidiaries etc. This mixed organizational culture may be an effect of mergers between organizations. Note also that organizational culture may be amplified by the official decision-making structure. If the organizational culture seems to be authoritative, do not use the participative approach to EM. Try other approaches such as e.g. interviewing.

If the organizational culture is undecided, try to negotiate that modeling will be done in two steps. The first step will function as an initial test of whether or not a participative approach is suitable. If active participation is not achieved in the modeling team, use traditional interviewing for the remainder of the project. If the culture is mixed, use the participative approach for work in the consensus-oriented part of the organization and some non-participative approach in the more authoritative parts of the organization. However, do not mix the two groups of people.

The organizational culture at KA is characterized by distributed working groups from different cultures as design and manufacturing facilities around the world require an awareness of how to integrate different ways of working. This formed an excellent basis for a consensus oriented way of working where inclusion of different opinions and equality regarding expressing contradictory viewpoints was accepted as a natural way of working. In the RCC the culture was mixed. Some organizational units had an authoritative culture and some had a consensus oriented culture. For the trial applications we chose three with consensus oriented culture.

In the SKaS case the culture was mixed. In general, the sense of hierarchy between different professions such as doctors and nurses in a healthcare organization is very strong. However, in the modeling group, where both doctors and nurses were represented, the culture was consensus oriented. This could be explained by the strong common dedication to solve the problem at hand.

Hidden agendas will decrease the possibility of achieving the project goals, since different stakeholders will try to steer the project towards their own goals. The project definition states the official goals of the project. It serves as important input to detecting hidden agendas. If the organization has hidden agendas, it may be reluctant to give the necessary authority to stakeholders, which could be "suspected" of jeopardizing that agenda. There can be hidden agendas as a part of a project, and the whole project itself could be a hidden agenda. The latter is the most fatal one.

Interviews with stakeholders before starting the project may reveal hidden agendas, but in that case they need to be carried out by an experienced person with good social 
skills. Questions about how the project was initiated, how the project is anchored in the organization and how the result will be used afterwards are useful as probes. Hidden agenda may also and will often surface during the project, which calls for open discussions with the customer.

In RCC the project itself did not have hidden agendas, but on the other hand it dealt with an issue - knowledge sharing - which related to some hidden agendas mostly concerning reluctance of information sharing. We identified these issues during interviews and as a result prepared modeling objectives addressing them.

In the SKaS case there were no hidden agendas since all stakeholders had complete agreement with regard to both the problem definition and the problem solution.

\subsection{Assess the Problem at Hand}

There are two views among practitioners when it comes to defining the problem at hand. Some stress the importance of obtaining a clear problem definition and seem to believe that it is possible to acquire such a clear definition. Others, often the most experienced practitioners, claim that clearly defined problems in most cases are illusions and that they rather are detected as the project progresses. The objective of the project is negotiated with the customer or process/project owner. There are two main approaches to this end - (1) interview the key decision maker(s) on the customer side about the objective, or (2) conduct short participative EM session to identify the objective, preferably involving other stakeholders than the key decision maker(s). Approach (2) can be used when it is difficult for the customer to pinpoint the problem, which normally means that the customer is uncertain about what exactly she/he wants to achieve. If the uncertainty still remains after the short EM session, this may indicate that the problem at hand is a "wicked problem".

Assessing the complexity of a problem definition is an essential part of the project negotiation. Problem complexity influences the project planning in terms of activities and resources. For resources, the complexity of the problem influences the requirements of the actor/s responsible for carrying out the EM project. Three types of problems can be observed:

- Fairly "simple" problems have a clear definition and a perceivable solution, and which do not require the co-ordination of a large number of different preconditions, activities, actors and resources.

- "Complex" problems have a fairly clear definition and a perceivable solution, but which require the co-ordination of a large number of different preconditions, activities, actors, and resources.

- "Wicked problems" are ill-structured problems, which have no clear problem definition and where there is no way of measuring that the problem is solved.

For "simple" and "complex" problems, proceed to the planning phase. If the problem is considered to be "complex", ensure that a highly skilled person will lead the project. If the problem is considered to be "wicked", negotiate that the project will be carried out in three steps:

1. A pre-study phase where modeling is the approach to obtaining agreement to the main scope of the project. 
2. A negotiation phase, where the actual project is negotiated and planned. Since a "wicked" problem comprises many unknown factors, the customer must be made aware of this. Preferably, the project planning should contain a number of evaluation steps, where the results of the project are evaluated and new decisions are made regarding the continuation of the project.

3. A completion phase, where the defined problem is solved as best can be done.

In the KA case, interviews with the project owner and the manager of the department under consideration gave a good impression of the problem at hand: the process of innovation had to be refined and probably restructured. As the complexity of this task even after the interviews was not fully clear, the decision was made to conduct several EM phases addressing different scopes and levels of detail. The selected C3S3P approach supported this intention quite nicely. However, it has to be noted that time plan and budget of the EU project MAPPER provided adequate frame conditions in terms of resources. In a commercial project, the frame conditions would have been subject of negotiation after every phase.

In the RCC case the project was initially seen as complex and the problem as unclear. After pre-interviewing (see section 3.2) the overall vision of KM at RCC and project's focus was modeled in two sessions with top management representatives. Based on the outcome of these sessions we planned the pilot cases.

In the SKaS case the problem was perceived to be fairly "simple" at the outset of the project. This assumption was not changed during the project. Hence, there was no need for re-planning.

\subsection{Assign Roles in the Modeling Process}

We recommend assigning the typical roles used in project management such as project owner, steering group and quality manager and in addition the following roles specifically related to participative EM projects.

The modeling facilitator is responsible for choosing the modeling language used in the sessions, conducting modeling sessions, assisting the modeling participants to discuss, capture and structure ideas, as well as helping to develop the model during the session. The facilitator is only there to moderate the problem solving process among the domain experts, not to solve the problem. The ownership of a problem and its solution should always remain with the stakeholders. We recommend using two modeling facilitators if possible. Two facilitators should always be used if the modeling group is larger than 8 people and/or if the duration is planned for more than 6 hours. In smaller projects the modeling facilitator may also act as tool operator.

The tool operator is responsible for drawing the model into a computerized tool. This can be done either after the modeling session or during the session. In the latter case the tool operator has to work in tandem with the facilitator to ensure that all the ideas and wishes of all participants are reflected in the model correctly.

The modeling participants, also called domain experts, are responsible for providing correct knowledge about the problem domain and making sure that it is reflected in the model. They are the problem solvers.

Allocating the relevant domain competency profiles to the project is a task that should not be taken lightly, since domain knowledge is the most critical resource in an EM project. One way of ensuring that the participating domain experts will contribute 
their knowledge in the modeling sessions is to interview them in advance. They also need to be prepared for what will happen during the sessions. This is particularly critical in organizations where the employees are not used to modeling in general and particularly to modeling in a group. Before the modeling session each participant has to: (1) understand the objective of the modeling session, (2) agree upon the importance of this objective, (3) feel personally capable to contribute to a positive result, and (4) be comfortable with the rest of the team (including the facilitator).

The best way of preparing the participants is to carry out individual interviews. In general, experienced facilitators do not face problems to get the customer to accept interviewing the modeling participants in advance as part of the project. In contrast, the less experienced claim that they seldom are given the opportunity to carry out interviews. They are in general aware of the importance of interviews and say that they need to improve their ability to negotiate the resources to carry them out.

At KA, interviews with the participants were done in a series of meetings with 2-3 persons. In these meetings, the area under consideration was discussed in order to prepare for the EM sessions and to investigate whether additional stakeholders should contribute specific domain knowledge. One result of these meetings was a textual description of the issues addressed. Furthermore, all participants of the EM sessions got basic training in the visual modeling language used and in the modeling tool.

In the RCC case the modeling participants were interviewed before the modeling session, which allowed us to plan the session, e.g. identify specific objectives, select participants, questions, and plan the course of the seminar.

In the SKaS case the participants in the modeling group were used to participative modeling from a previous project and stated positive experiences from that. The previous project had the same modeling facilitator. The participants in the group had all been involved in defining the project. Therefore, the project leader decided that interviewing the participants was not necessary. The positive outcome of the project supported this view.

The competency of the others than domain experts is equally critical. Three aspects of the project determine the needed EM competency in the team of method experts:

1. The degree of problem complexity. A wicked problem will need a more experienced and skilled modeling team than a simple problem.

2. The degree of creativity needed for the solution. Designing the future state or radically changing the current state needs more method competency than describing the current state.

3. The size of the project and the needed co-ordination effort. A large modeling project will need a leader and experienced and skilled method expert with a holistic view. Furthermore, a large project might also require someone being responsible only for documenting and managing the modeling results.

In the RCC case the problem was complex and the problem definition was abstract allowing many alternative solutions involving different stakeholder types. To answer these challenges three pilot projects addressed the problem from different perspectives. They each had a pilot owner, a modeling facilitator and a tool operator. The owner of the whole project at RCC coordinated the efforts of the pilot cases. In summary the complexity and the size of the project required experienced modeling facilitators with project management skills. 
In the SKaS case the problem was fairly "simple" and the project definition gave very little need for creativity. The size of the project when it comes to modeling activities was rather small. Since the project leader and facilitators were experienced the project was carried out in a very controlled manner.

\subsection{Acquire Resources for the Project in General and for Preparation Efforts in Particular}

One important insight that characterizes experienced EM practitioners is that it is unprofessional to assume responsibility for a project without the necessary resources [11]. A professional attitude, although drastic, is to refuse projects without the proper resources. This seems in fact to be part of the professional ethics of expert practitioners.

Management support is essential for a project to be successful. It is a critical precondition for obtaining the necessary resources and for motivating stakeholders to commit to the modeling work. It will also facilitate the involvement of skilled method experts even if it may be costly. Management should also give the modeling team the authority to act and make decisions within the boundaries of the project. A critical issue is the persistence with which management keeps supporting the project even if more resources are needed later on, or if the project runs into other types of problems. This is particularly important if the project is larger than 1-2 modeling sessions.

The KA case is an excellent example for the positive effects of full management support. After having made the decision to conduct the project, which was based on a clear description of goals and the planned process, the responsible manager did not only arrange for the required personnel resources and facilities, but also promoted the project in the organization and opened all desirable information sources.

In the RCC case management provided enough resources to carry out the pilot applications. On the other hand the top management representatives sometimes canceled their participation at a modeling seminar or sent a replacement instead.

In the SKaS case support from management was strong. The modeling team was given the needed modeling resources and the authority to carry out the project as they deemed fit. This shows that management had great confidence in the team and that the priority of the project was high.

Our case studies and interview study indicate that the effort spent on preparation is in direct relation to the quality of the project results. Experienced practitioners claim that they do not accept projects where the resources for preparation are too small, while the inexperienced report severe problems that are related to lacking preparation. We suggest distributing effort in a modeling project according to: preparations (assessing the organization, project definition, interviews, etc) $\sim 40 \%$, modeling seminars $\sim 30 \%$, and documenting and reporting $\sim 30 \%$ of the total effort. The figures are mainly based on interviews with practitioners with 10-30 years of participative EM experience. This distribution of resources is only given as an indication; depending on the project aim and duration they may actually vary within ca $10 \%$. E.g. some very short projects might not require extensive documentation.

The KA and RCC cases followed this distribution of effort in general. In the SKaS case the distribution of effort was, however, different. The need for preparation was much smaller due to the fact that the problem was simple and well defined and that 
the modeling team knew each other from before. Also, the domain experts in the modeling group were involved in defining the project.

\subsection{Conduct Modeling Sessions}

Carrying out a modeling session needs concentration and dedication from all participants involved. A detailed discussion about how a session should be managed is beyond the scope of this paper. However, we consider the following issues to be of utmost importance for the quality of the outcome of a modeling session:

- Each modeling session should have set clear objectives of practical value to the organization.

- Use a modeling notation that everyone understands. Participation will be severely hampered if too much attention is put into understanding the notation used. If the participants are not used to modeling, use a relatively simple and intuitive notation.

- Do not "train" the modeling participants in method knowledge. It is the responsibility of the modeling facilitator that the chosen method/notation is correctly used. Too much attention to the method/notation used will distract the modeling participants from solving the problem at hand. Our experience is that hands-on practice is the best way of becoming acquainted with a method/notation.

- Keep everyone involved and focused on the problem at hand. Avoid side discussions that will distract attention from the problem at hand.

- Do not accept unknown participants in the modeling session. In the best case they will keep silent and leave early, because they do not have the background knowledge that allows them to participate efficiently. In the worst case they might try to sabotage the modeling effort, to fulfill their own agenda - something that should have been discovered in the pre-interviewing stage.

- The problem owner and/or the insiders of the problem area should not dominate the seminar - the point of having a broader modeling group is to extend the view.

- Establish a common vocabulary - developing a CM might help to achieve this.

- Develop models in parallel - e.g. decide on a business goal, then switch to modeling a business process that would fulfill the goal, and then model the necessary roles performing and being responsible for the process. How to shift the group's attention between the sub-models depends on the project objectives, the situation in the organization, and the findings in the pre-interviews. More guidance about this is available in [6] and [11].

- Make concrete decisions in the session - attach roles and responsibilities to goals, processes and change actions.

- The same model might need to be improved in several modeling sessions because the group's understanding of the modeling issue tends to change during the project.

- The result of the session, the model, should deliver a solution - a common situation is that the model is too "polite", addressing only general and well known issues without tackling some of the hard problems of the organization.

- Make sure that everyone knows what will happen after the seminar - and whether they should carry out some of the actions decided and documented in the model. 
In the RCC case we followed these guidelines. On a few occasions of top level managers sent replacements to modeling seminars. These people were either unable to contribute and left early or started to investigate how is this project related to other projects which they knew.

In the SKaS case modeling successfully proceeded according to the above recommendations. Since the participants were familiar with modeling from before, there was need to train them. In the previous project they were involved, however, there was a conscious decision to train the participants by hands on experience, which proved successful. The EKD notation was perceived to be easy to understand by the participants, even if they had no previous experience from modeling. The modeling team was stable throughout the project, which facilitated the constant refinement of the EKD concepts model, which was at the center of the modeling activities.

\section{Concluding Remarks}

This paper has presented a number of generic recommendations for carrying out participative EM in diverse organizational contexts. The main elements of EM are the notation and the modeling process. In participative EM, most of the critical success factors pertain to the modeling process. The positive effects of participative EM are: (1) Enhanced quality of the Enterprise Model, (2) Consensus among stakeholders, and (3) Acceptance and commitment to the modeling result [11]. However, to achieve these effects substantial knowledge and understanding of the modeling process is needed, as well as experience and skills with regard to managing people in a modeling session. Hence, successfully carrying out participative EM is a task that is far from trivial, which requires skillful and experienced professionals both when it comes to the roles of facilitator and project manager. In summary, this emphasizes the need for developing effective training programmes for facilitators and EM project managers.

\section{References}

1. Persson, A., Stirna, J.: An explorative study into the influence of business goals on the practical use of Enterprise Modelling methods and tools. In: Proceedings of the 10th International Conference on Information Systems Development (ISD 2001), Kluwer, London (2001)

2. Rittel, H.W.J., Webber, M.M.: Planning Problems are Wicked Problems. In: Cross (ed.) Developments in Design Methodology, John Wiley \& Sons, Chichester (1984)

3. F3-Consortium. F3 Reference Manual, ESPRIT III Project 6612, SISU, Sweden (1994)

4. Loucopoulos, P., Kavakli, V., Prekas, N., Rolland, C., Grosz, G., Nurcan, S.: Using the EKD Approach: The Modelling Component, UMIST, Manchester, UK (1997)

5. Nilsson, A.G., Tolis, C., Nellborn, C. (eds.): Perspectives on Business Modelling: Understanding and Changing Organisations. Springer-Verlag, Heidelberg (1999)

6. Bubenko, J.A., j., P.A., Stirna, J.: User Guide of the Knowledge Management Approach Using Enterprise Knowledge Patterns, IST Programme project Hypermedia and Pattern Based Knowl-edge Management for Smart Organisations, no. IST-2000-28401, KTH, Sweden, (2001) http://www.dsv.su.se/ js/ekd_user_guide.html 
7. Niehaves, B., Stirna, J.: Participative Enterprise Modelling for Balanced Scorecard Implementation. In: 14th European Conference on Information Systems (ECIS 2006), Gothenburg, Sweden (2006)

8. Stirna, J., Persson, A., Aggestam, L.: Building Knowledge Repositories with Enterprise Modelling and Patterns - from Theory to Practice. In: proceedings of the 14th European Conference on Information Systems (ECIS), Gothenburg, Sweden (June 2006)

9. Carstensen, A., Högberg, P., Holmberg, L., Johnsen, S., Karlsen, D., Lillehagen, F., Lundqvist, M., Ohren, O., Sandkuhl, K., Wallin, A.: Kongsberg Automotive Requirements Model, deliverable D6, MAPPER, IST proj. no 016527 (2006)

10. Mikelsons, J., Stirna, J., Kalnins, J.R., Kapenieks, A., Kazakovs, M., Vanaga, I., Sinka, A., Persson, A., Kaindl, H.: Trial Application in the Riga City Council, deliverable D6, IST Programme project Hypermedia and Pattern Based Knowledge Management for Smart Organisations, project no. IST-2000-28401. Riga, Latvia (2002)

11. Persson, A.: Enterprise Modelling in Practice: Situational Factors and their Influence on Adopting a Participative Approach, PhD thesis, Dept. of Computer and Systems Sciences, Stockholm University, No 01-020, (2001) ISSN 1101-8526

12. Willars, H.: Handbok i ABC-metoden. Plandata Strategi (1988)

13. Bajec, M., Krisper, M.: A methodology and tool support for managing business rules in organisations. Information Systems 30(6), 423-443 (2005)

14. Castro, J., Kolp, M., Mylopoulos, J.: A Requirements-Driven Software Development Meth-odology. In: CAiSE 2001. LNCS, vol. 2068, pp. 108-123. Springer, Heidelberg (2001)

15. Dobson, J., Blyth, J., Strens, R.: Organisational Requirements Definition for Information Technology. In: Proceedings of the International Conference on Requirements Engineering 1994, Denver/CO (1994)

16. Fox, M.S., Chionglo, J.F., Fadel, F.G.: A common-sense model of the enterprise. In: Proceedings of the 2nd Industrial Engineering Research Conference, Institute for Industrial Engineers, Norcross/GA (1993)

17. Yu, E.S.K., Mylopoulos, J.: From E-R to A-R - Modelling Strategic Actor Relationships for Business Process Reengineering. In: Proceedings of the 13th International Conference on the Entity-Relationship Approach, Manchester, England (1994)

18. Zorgios, Y., (ed.): Enterprise State of the Art Survey, Part 3, Enterprise Modelling Methods, DTI ISIP Project Number 8032, AIAI, The University of Edinburgh (1994)

19. Krogstie, J., Jørgensen, H.D.: Interactive Models for Supporting Networked Organizations. In: Proceedings of CAiSE'2004. LNCS, Springer, Heidelberg (2004)

20. Krogstie, J., Lillehagen, F., Karlsen, D., Ohren, O., Strømseng, K., Thue Lie, F.: Extended Enterprise Methodology. Deliverable 2 in the EXTERNAL project, available at (2000) http://research.dnv.com/external/deliverables.html

21. Lillehagen, F.: The Foundations of AKM Technology. In: Proceedings 10th International Conference on Concurrent Engineering (CE), Madeira, Portugal (2003) 